\title{
Proton Transfer in Proton Glasses
}

\section{Authors: V. Hugo Schmidt}

NOTICE: this is the author's version of a work that was accepted for publication in Journal of Molecular Structure. Changes resulting from the publishing process, such as peer review, editing, corrections, structural formatting, and other quality control mechanisms may not be reflected in this document. Changes may have been made to this work since it was submitted for publication. A definitive version was subsequently published in Journal of Molecular Structure, [VOL\# 177, (July 1, 1988)] DOI\#10.1016/0022-2860(88)80092-9

Schmidt, V. Hugo. "Proton Transfer in Proton Glasses." Journal of Molecular Structure 177 (July 1988): 257-264. doi:10.1016/0022-2860(88)80092-9.

Made available through Montana State University's $\underline{\text { ScholarWorks }}$ scholarworks. montana.edu 


\title{
PROTON TRANSFER IN PROTON GLASSES
}

\author{
V. HUGO SCHMIDT \\ Physics Dept., Montana State University, Bozeman, MT 59717 (U.S.A.) \\ (Received 14 September 1987)
}

\begin{abstract}
Proton intrabond transfer in $\mathrm{O}-\mathrm{H} \cdots \mathrm{O}$ bonds is the mechanism for dynamic behavior of rubidium/ammonium dihydrogenphosphate mixed crystals. Because of strong proton-proton interactions, such transfer is associated with thermally activated creation of $\mathrm{H}_{3} \mathrm{PO}_{4}-\mathrm{HPO}_{4}$ intrinsic defect pairs, their hindered diffusion in a random-step fractal potential, and their eventual annihilation. Predictions of a model based on this behavior are compared with determinations on a $35 \%$ ammoniated crystal by Courtens et al. of dielectric susceptibility and of relaxation time distribution limits calculated from Brillouin scattering results.
\end{abstract}

The proton glass [1] $\mathrm{Rb}_{1-x}\left(\mathrm{NH}_{4}\right)_{x} \mathrm{H}_{2} \mathrm{PO}_{4}$ (RADP) is a mixed crystal of $\mathrm{RbH}_{2} \mathrm{PO}_{4}(\mathrm{RDP})$ and $\mathrm{NH}_{4} \mathrm{H}_{2} \mathrm{PO}_{4}(\mathrm{ADP})$. $\mathrm{RDP}$ and $\mathrm{ADP}$ have the same paraelectric $(\mathrm{PE})$ phase structure. They undergo ferroelectric $(\mathrm{FE})$ and antiferroelectric (AFE) order-disorder transitions at 146 and $148 \mathrm{~K}$ respectively which order the "acid" $\mathrm{O}-\mathrm{H} \cdots \mathrm{O}$ protons differently. RADP has no transitions for $0.22<x<0.74$, but proton glass (PG) behavior exhibiting a wide spread in relaxation times sets in near $75 \mathrm{~K}$. This behavior is described accurately by a phenomenological model [1] with adjustable parameters based on the VogelFulcher law.

The present work analyzes this dynamic behavior with a microscopic model having essentially no adjustable parameters. It is based on two concepts: (1) creation and annihilation of mobile intrinsic defects, and (2) hindered diffusion of these defects in a random potential.

Brawer [2] discussed similar concepts in relation to structural glasses. In the PG system the defects and their creation and diffusion energies are better understood. Spin and quadrupolar glasses, on the contrary, do not seem to exhibit relaxation caused by diffusing defects.

Static behavior of RADP including the $x-T$ phase diagram was predicted [3] by letting the Slater interaction $\epsilon_{0}$ which causes the FE transition in RDP be independent of $x$, and letting the cross-cation interaction $\epsilon_{a}$ which causes the AFE transition in ADP be proportional to $x$ in that mean-field model. 
Dynamic behavior requires acid proton intrabond transfers associated with effective diffusion of thermally generated intrinsic $\mathrm{HPO}_{4}$ and $\mathrm{H}_{3} \mathrm{PO}_{4}$ "Takagi" defects of energy $\epsilon_{1}$. All these proton-proton interactions can be represented by pseudospin interactions [4].

Glassy systems have structural randomness which causes frustration and dynamic disorder. In RADP the random placement of $\mathrm{Rb}^{+}$and $\mathrm{NH}_{4}^{+}$cations causes competing FE and AFE interactions leading to frustration which prevents FE or AFE ordering. Instead, the acid proton system has dynamic disorder and in the $\mathrm{PG}$ regime develops a wide spread in relaxation times characteristic of glassy systems.

A microscopic model for proton glass dynamics thus must deal with the dynamics of the acid proton system. Presented here is the first such analytic model. Its predictions are compared with dielectric and Brillouin scattering experimental results.

The flow chart below outlines the steps in the model calculation.

$$
\left.\begin{array}{r}
\tau_{0} \\
\epsilon_{d} \rightarrow U(r) \\
\left(D, \epsilon_{1}\right)
\end{array}\right\}\left\{\begin{array}{r}
\rightarrow R(t) \\
\rightarrow\left(R_{0}, n\right)
\end{array}\right\} \rightarrow P(t) \rightarrow\left(\epsilon^{\prime}, \epsilon^{\prime \prime}\right)
$$

Following the flow chart from left to right, $\epsilon_{d}$ is the r.m.s. defect diffusion energy. These Takagi $\mathrm{HPO}_{4}$ and $\mathrm{H}_{3} \mathrm{PO}_{4}$ defects are considered as the moving entities rather than individual protons because the strong proton-proton interactions cause proton motions to be highly correlated.

Creation and annihilation of Takagi defects involve the processes

$$
\mathrm{H}_{2} \mathrm{PO}_{4}+\mathrm{H}_{2} \mathrm{PO}_{4} \leftrightarrow \mathrm{H}_{3} \mathrm{PO}_{4}+\mathrm{HPO}_{4}
$$

Their effective diffusion via the processes

$$
\mathrm{H}_{3} \mathrm{PO}_{4}+\mathrm{H}_{2} \mathrm{PO}_{4} \leftrightarrow \mathrm{H}_{2} \mathrm{PO}_{4}+\mathrm{H}_{3} \mathrm{PO}_{4} \text { and } \mathrm{HPO}_{4}+\mathrm{H}_{2} \mathrm{PO}_{4} \leftrightarrow \mathrm{H}_{2} \mathrm{PO}_{4}+\mathrm{HPO}_{4}
$$

simply requires $\mathrm{O}-\mathrm{H} \cdots \mathrm{O} \leftrightarrow \mathrm{O} \cdots \mathrm{H}-\mathrm{O}$ intrabond proton transfer. The importance of Takagi groups for proton glass dynamics is generally recognized [4-6].

To find $\epsilon_{d}$ we examine the pseudospin system Hamiltonian $H$. Kinetic energy terms decribing pseudospin energy exchange with the phonon bath are omitted. Hence $H$ is equivalent to the internal energy $U$ given by

$$
\begin{array}{r}
H=U=N\left\{n\left(\epsilon_{1}-R \frac{b}{b} \epsilon_{d}\right)+(1-f) \epsilon_{0}\right\}+\sum_{i}\left(\epsilon_{a} / 2\right) S_{i}\left[S _ { j } \delta \left(s_{i j}\right.\right. \\
\left.-1)+S_{k} \delta\left(s_{i k}-1\right)\right]
\end{array}
$$

Here $N$ is the number of molecular units. The first term will be considered later. In the second term, $(1-f)$ is the fraction of units having Slater energy 
$\epsilon_{0}$. In the third term, $j$ and $k$ indicate the protons with which proton $i$ interacts across $\mathrm{NH}_{4}^{+}$ions only, (see ref. 4) because $s_{i j}$ and $s_{i k}$ are +1 and -1 for $\mathrm{NH}_{4}^{+}$and $\mathrm{Rb}^{+}$ions respectively, located between the $i$ and $j$ or $i$ and $k$ protons. The sum does not count $S_{i} S_{j}$ or $S_{i} S_{k}$ interactions twice. The $\epsilon_{1}$ and $\epsilon_{0}$ terms could also be written in terms of pseudospin interactions [4], but the above form is more useful in finding $\epsilon_{d}$.

The energy change $\epsilon$ per step has contributions only from the $\epsilon_{a}$ and $\epsilon_{0}$ interactions. The mean square change contributions per step are additive and are given by

$\epsilon_{d}^{2}=\left\langle(\epsilon)^{2}\right\rangle=2 x \epsilon_{a}^{2}+2 f_{g}\left(1-f_{g}\right) \epsilon_{0}^{2}$

Here $x=0.35$ is the ammonium fraction and $f_{g}=0.77291$ is the limiting fraction [3] of zero-energy Slater groups in the PG regime. The first term shows that in pure $\mathrm{ADP}$ a proton transfer has probability $\frac{1}{2}$ each of changing $U$ by $\pm 2 \epsilon_{a}$ and of leaving $U$ unchanged. The second term assumes that the probability of creating or annihilating a Slater group of energy 0 or $\epsilon_{0}$ is proportional to the respective fractions $f_{g}$ and $1-f_{g}$ of those groups already present. Because $\epsilon_{a}$ as defined in eqn. (1) and ref. 3 equals $\epsilon_{0}$ (and not $2 \epsilon_{0}$ as stated in refs. 3 and 4 ), and because the best value known [7] for $\epsilon_{0} / k$ is $74.5 \mathrm{~K}$ which applies to pure RDP, we obtain the value $\epsilon_{d}=76.4 \mathrm{~K}$.

The $\epsilon$ distribution leading to eqn. (2) is discrete but nearly equivalent to the normalized gaussian form

$w(\epsilon)=(2 \pi)^{-\frac{1}{2}} \epsilon_{d}^{-1} \exp \left[-\left(\epsilon-\epsilon_{\mathrm{b}}\right)^{2} / 2 \epsilon_{d}^{2}\right]$

The bias energy $\epsilon_{\mathrm{b}}$ in thermal equilibrium must be such that defect diffusion on the average does not change $U$.

To find the distribution $W(\epsilon)$ of internal energy change $\epsilon$ per step along the paths actually taken, we make the approximation that

$$
W(\epsilon)=w(\epsilon) \exp (-\epsilon / k T)
$$

Then the assumption that $U$ is unbiased along paths actually taken fixes the value of $\epsilon_{\mathrm{b}}$ and gives $W(\epsilon)$ the simple form

$$
W(\epsilon)=(2 \pi)^{-\frac{1}{2}} \epsilon_{d}^{-1} \exp \left(-\epsilon^{2} / 2 \epsilon_{d}^{2}\right) \text { if } \epsilon_{\mathrm{b}}=\epsilon_{d}^{2} / k T
$$

If we ignore motion of other Takagi defects we can equate $U$ to an internal energy potential $U(r)$ of a defect $r$ steps along its diffusion path away from its original site. Although $U(r)$ defined in this way is single-valued, $U$ for a given defect at a given site can be multivalued because the change in $U$ in going to a new site depends on the path. The $2-d$ analog of this potential is a sinkhole with caves in the surrounding slopes, so that though an outward path (A in Fig. 1) chosen randomly using $w(\epsilon)$ is uphill, a typical actual outward path (B in Fig. 1) using $W(\epsilon)$ is level on the average.

The crystal topology [4] is such that the defect can diffuse around a loop of 


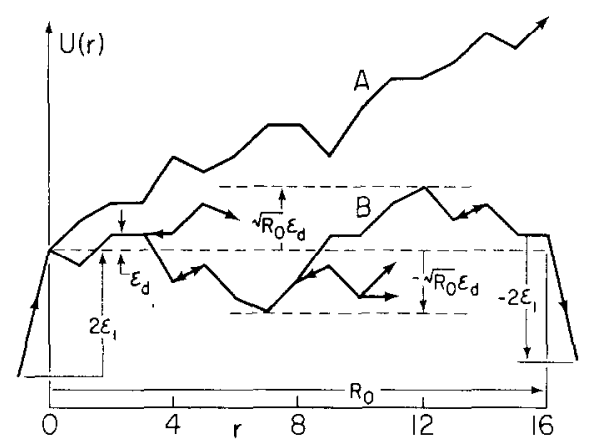

Fig. 1. Typical Takagi defect diffusion paths illustrating internal energy $U(r)$ plotted vs. number of steps $r$ from defect creation site. Here $\mathrm{A}$ is a path employing the biased $w(\epsilon)$ step height distribution of eqn. (3), while B is an actual path with side branches using the unbiased $W(\epsilon)$ of eqn. (5). Also shown are the defect pair creation and annihilation energies $\pm 2 \epsilon_{1}$, the r.m.s. diffusion step height $\epsilon_{d}$, and the assumed maximum barrier and trapping energies $\pm R_{\delta}^{b} \epsilon_{d}$, where $R_{0}$ is the number of steps from creation to annihilation with a new partner.

6 (or 8,10 , etc.) steps back to its original position. The importance of these loops is estimated by a statistical analysis. A defect can take one of six 6-step loops or one of ten 8 -step loops to return to its original site. For each step there is approximate probability $1 / 3$ of visiting the correct new site on a given loop, giving probability $2 / 243$ of traversing some loop 6 steps long, and 10/6561 for a loop 8 steps long, without taking side trips. Backtracking is allowed, and side trips are few because the diffusion path Hausdorff dimensionality $D$ is near 1 as discussed below, so traversing of loops appears unimportant.

The topology of the diffusion paths available to a defect is locally equivalent to a double-branching Cayley tree because a defect can move to two new positions or return to its former site. Because looping is unimportant we studied diffusion on such a Cayley tree for a number of paths of maximum length $r=13$ steps from the origin, using a Monte Carlo technique. These simulations indicate that the number $N_{\mathrm{s}}$ of new sites visited obeys the fractal relation $N_{\mathrm{s}}=r^{D}$, with Hausdorff dimensionality [8] $D=1.15 \pm 0.05$. A value 1 for $D$ would correspond to one-dimensional $(1-d)$ diffusion with no side trips.

Although a Takagi defect occasionally takes a branch path as shown in Fig. 1 , upon retracing it the polarization changes made while taking that branch are cancelled. Thus the net path causing polarization change is at any instant strictly $1-d$. Because $U(r)$ along such $1-d$ paths obeys the unbiased $\epsilon$ distribution of eqn. (5), $U(r)$ is a fractal potential of a type considered by Dotsenko [9].

The time per diffusion step depends on the Boltzmann factors found from $U(r)$ for the defect's three possible new sites, and on the attempt time $\tau_{0}$. Brillouin scattering studies by Courtens et al. [10] fix $\tau_{0}$ at $5 \times 10^{-14} \mathrm{~s}$.

The net effect of diffusion over a time interval $t$ is proton shifts only along 
a $1-d$ path of diffusion path length $R(t)$ steps. The maximum barrier encountered in $R$ steps for the $W(\epsilon)$ distribution of eqn. (5) is about $\epsilon_{d} R^{\frac{1}{2}}$ as shown in Fig. 1 , where $\frac{1}{2}$ is Dotsenko's scaling exponent $\nu$. This maximum barrier is unlikely to occur in a branch path, because if it did, the defect would have to cross it twice.

At high temperature the barriers are negligible, so the defect jump time is simply $\tau_{0}$ and it requires a time near $\tau_{0} R^{2}$ [Dotsenko's eqn. (11)] to diffuse $R$ steps. But in the PG regime the largest barrier dominates the diffusion time, so the factor $R^{2}$ should be omitted, giving for $\nu=\frac{1}{2}$ a diffusion time in agreement with Dotsenko's eqn. (9), which is also given below for arbitrary $\nu$

$$
\begin{aligned}
& t(R)=\tau_{0} \exp \left(\epsilon_{d} R^{\frac{1}{2}} / k T\right) \\
& R(t)=\left\langle R^{2}\right\rangle^{\frac{1}{2}}=\left[\left(k T / \epsilon_{d}\right) \ln \left(t / \tau_{0}\right)\right]^{1 / \nu}
\end{aligned}
$$

(Dotsenko's eqn. (9) - here $1 / \nu=2$ ). This equation for $1-d$ systems is applicable because the Takagi defect diffusion path is close enough to being $1-d$ so that the maximum barrier is unlikely to be in a branch path.

One-dimensional discrete-time random walks with random transition probabilities $p$ have been treated by several authors. In particular, the probability distribution

$\rho(p)=\left[\delta\left(p-p_{+}\right)+\delta\left(p-p_{-}\right)\right] / 2, p_{ \pm}=\exp \left( \pm \epsilon_{d} / 2 k T\right) / 2 \cosh \left(\epsilon_{d} / 2 k T\right)$

corresponding to a potential having random steps $\pm \epsilon_{d}$ from site to site (which gives the same results for our model as the $W(\epsilon)$ distribution in eqn. (5)) is a special case of a distribution presented by Sinai [11] and discussed by Derrida and Pomeau [12] which leads to the above $\ln ^{2}(t)$ dependence of $\left\langle R^{2}\right\rangle^{\frac{1}{2}}$, as does a more general random potential with a distribution of $E_{d}$.

The fractional defect density $n$ for Takagi defects of energy $\epsilon_{t}$ is found from the Boltzmann factor for such groups, noting that the a priori (infinite $T$ ) concentration of Takagi groups is $\frac{1}{2}$, and using the fact that $n \ll 1$ :

$n=\exp \left(-\epsilon_{t} / k T\right) /\left[1+\exp \left(-\epsilon_{t} / k T\right)\right] \simeq \exp \left[-\left(\epsilon_{1}-R_{b}^{\frac{1}{2}} \epsilon_{d}\right) / k T\right.$

Here $\epsilon_{1}$ is the Takagi group creation energy for this mixed crystal, which should be close to the value $\epsilon_{1} / k=647 \mathrm{~K}$ found [7] for pure RDP. The average defect annihilation path length $R_{0}$ is the path length in steps from creation to annihilation with a new partner. The trapping energy $-R_{b}^{\frac{1}{d}} \epsilon_{d}$ shown in Fig. 1 is found by the same argument used above to find maximum barrier height $+R^{\frac{1}{2}} \epsilon_{d}$.

A second relation between $R_{0}$ and $n$ comes from the above relation between $N_{s}$ and $r$. We set $r=R_{0}$ and find $N_{s}$ by noting that the defect has probability near $n$ of annihilating with a new partner at each new site visited, so on the average it will visit $N_{s}=n^{-1}$ sites, giving the relation 
$n^{-1}=R_{0}^{D}$

An implicit relation for $n$ is found from eqns. (8) and (9)

$n=\left\{\left[\epsilon_{1}+k T \ln (n)\right] / \epsilon_{d}\right\}^{-2 D}$

The polarization relaxation $P(t)$ following step removal at $t=0$ of a small d.c. electric field is found by integrating the fractional polarization change $\mathrm{d} P$ / $P$ which equals $n \mathrm{~d} R(\mathrm{~d} t)$ if the defect wanders randomly, as at high temperature. Here $\mathrm{d} R(\mathrm{~d} t)$ is the mean number of $\mathrm{O}-\mathrm{H} \cdots \mathrm{O}$ dipoles reversed by a Takagi defect during time $\mathrm{d} t$. Near the FE transition temperature $T_{\mathrm{c}}$ for Rbrich crystals the defect path is non-random and the relation shows critical behavior

$\mathrm{d} P / P=-\left[\left(T-T_{\mathrm{c}}\right) T\right] n \mathrm{~d} R$

In the PG concentration range, $T_{c}$ can be approximated by 0 . Then upon integration, $\ln (P)+$ const $=-n R$. Taking the exponent and substituting for $R$ from eqn. (6) yields for decay from an initial polarization $P_{i}$ the expression

$P / P_{i}=\exp \left\{-n\left[\left(k T / \epsilon_{d}\right) \ln \left(1+t / \tau_{0}\right)\right]^{2}\right\}$

This "logarithmic gaussian" decay has $\ln ^{2}(t)$ in the exponent. A more general form with arbitrary power of $\ln (t)$ was derived by Dotsenko [9], and by Palmer et al. using two models. The first assumes hierarchically constrained dynamics [13], but with collaboration of several pseuodspins required to advance from one level to the next, rather than surmounting of random potential barriers. The second involves relaxation of isolated clusters of unfrustrated spins [14].

The conversion from step response in eqn. (12) to a.c. response is done by integrating the responses at time $t$ to an a.c. electric field of angular frequency $\omega$ made up of differential steps beginning at times $t^{\prime}<t$. The complex dielectric susceptibility $\epsilon=\epsilon^{\prime}-j \epsilon^{\prime \prime}$ determined in this way is

$\epsilon=\epsilon_{\infty}+\left(\epsilon_{\mathrm{dc}-\epsilon_{\infty}}\right)\left\{1-\int_{0}^{\infty}(\sin u+j \cos u) \exp \left[-a^{2} \ln ^{2}(1+u / f)\right] \mathrm{d} u\right\}$

where $u=\omega\left(t-t^{\prime}\right), a=k T n^{\frac{1}{2}} / \epsilon_{d}, f=\omega \tau_{0}$, and $\epsilon_{\mathrm{dc}}$ and $\epsilon_{\infty}$ are the $\epsilon^{\prime}$ values at temperatures just above and below the dispersion region.

We compare predictions of this model with dielectric results at 326 and 33700 $\mathrm{Hz}$ and with the relaxation time range found by a Brillouin scattering study at $\mathrm{GHz}$ frequencies. We use $\tau_{0}=5 \times 10^{-14} \mathrm{~s}$ as found by Courtens et al. [10] and $D=1.15$ as discussed above. For $\epsilon_{d}$ and $\epsilon_{1}$ we obtained best fit by using $50 \mathrm{~K}$ and $400 \mathrm{~K}$ instead of the values $76.4 \mathrm{~K}$ and $647 \mathrm{~K}$ expected from behavior of pure RDP and ADP as discussed above. Both fitting parameters are about $36 \%$ 

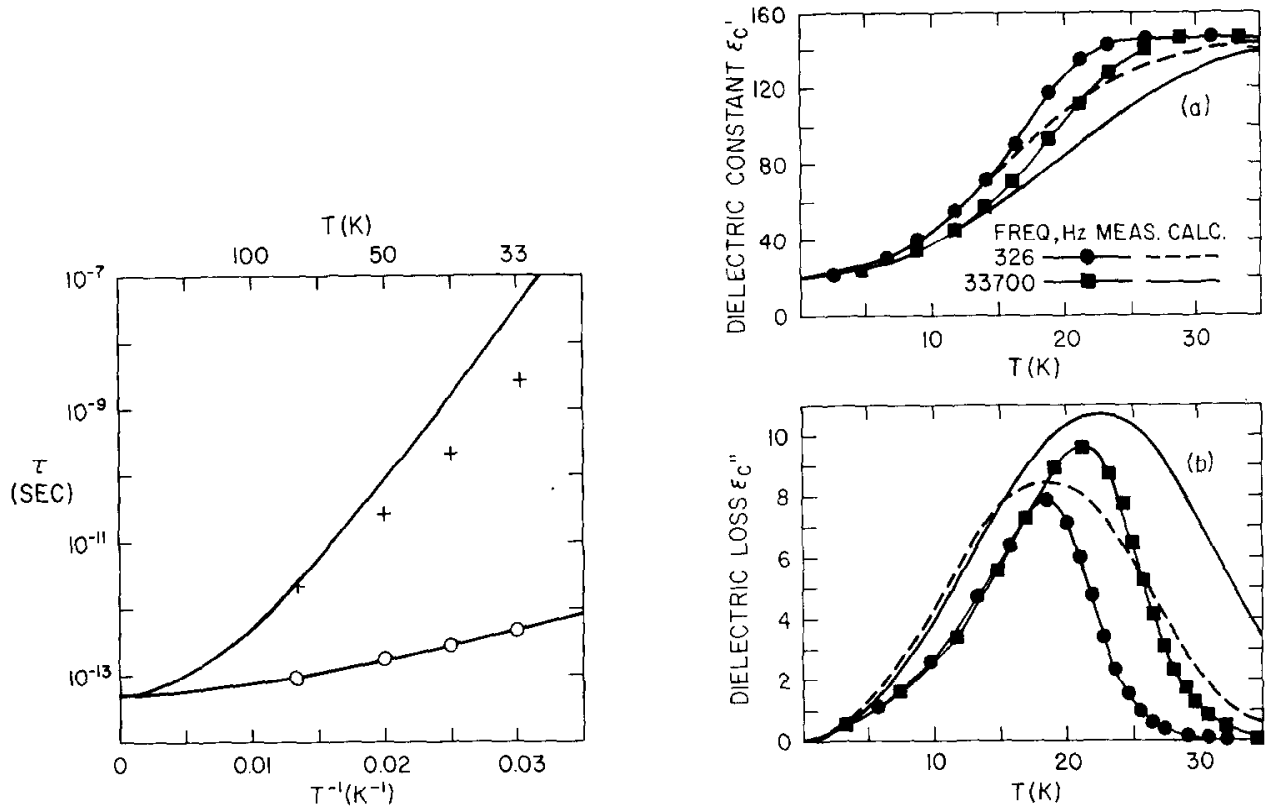

Fig. 2. Comparison of upper limits $\tau_{2}$ and lower limits $\tau_{1}$ of range of relaxation time $\tau$ having normalized weighting function $g(\tau)=\left[\tau \ln \left(\tau_{2} / \tau_{1}\right)\right]^{-1}$, where + and $O$ represent $\tau_{2}$ and $\tau_{1}$ determined by Courtens, Vacher and Dagorn [10] from Brillouin scattering measurements on $\mathrm{Rb}_{0.65}\left(\mathrm{NH}_{4}\right)_{0.35} \mathrm{H}_{2} \mathrm{PO}_{4}$, and solid lines are these limits predicted from eqn. (15) using $\tau_{0}=5 \times 10^{-14}$ $\mathrm{s}, D=1.15, \epsilon_{1}=400 \mathrm{~K}$, and $\epsilon_{d}=50 \mathrm{~K}$.

Fig. 3. Comparison of dielectric susceptibility measured in $\mathrm{Rb}_{0.65}\left(\mathrm{NH}_{4}\right)_{0.35} \mathrm{H}_{2} \mathrm{PO}_{4}$ by Courtens [1] with that calculated from eqn. (13) using parameters listed in Fig. 2.

below the expected values, so the fit for the expected parameters can be found by compressing the temperature scales in Figs. 2 and 3 by $36 \%$.

The expression for proton glass relaxation used [10] in analyzing Brillouin scattering results assumes a spread in relaxation time $\tau$ between $\tau_{1}$ and $\tau_{2}$ with normalized weighting function

$g(\tau)=\left[\tau \ln \left(\tau_{2} / \tau_{1}\right)\right]^{-1}$

Fitting magnitude and slope of the step function response for this $\tau$ distribution to that of eqn. (12) at the $\frac{1}{2}$ amplitude point yields

$\tau_{1}=\tau_{0} \exp \left(0.232 \epsilon_{d} / 2 n^{\frac{1}{2}} k T\right)$

$\tau_{2}=\tau_{0} \exp \left(1.433 \epsilon_{d} / 2 n^{\frac{1}{2}} k T\right)$

The relaxation time limits from eqn. (15) are compared in Fig. 2 to the values deduced by Courtens, Vacher and Dagorn [10] from Brillouin scattering experiments and shown in their Fig. 2. The model fits the lower time constant limit exactly, but somewhat overestimates the upper time constant limit. 
The comparison with a.c. dielectric susceptibility results of Courtens [1] using eqn. (13) is shown in Fig. 3. The predicted loss peaks occur at the right temperatures but are too broad, and the $\epsilon^{\prime}$ fits are not exact.

The overall comparison to Brillouin scattering and dielectric results covers 8 orders of magnitude in frequency and temperatures from 5 to $75 \mathrm{~K}$. The overall fit is quite satisfying, because of the four parameters in the model, only two had to be changed somewhat from their expected values.

Presented here are ideas concerning hindered diffusion of intrinsic defects and the corresponding dielectric response, and examples of diffusion in a fractal potential and of hierarchically constrained dynamics. These ideas may provide hints regarding relaxation via the complex diffusion mechanisms found in structural glasses.

\section{ACKNOWLEDGEMENTS}

Thanks are expressed to R. Blinc and S. Žumer for valuable discussions, to George Tuthill for formulating and Seth Cameron for carrying out the Takagi defect diffusion Monte Carlo calculation, and to E. Courtens for supplying preprints of his work. My stay at the Stefan Institute was supported by a Fulbright Fellowship.

\section{REFERENCES}

E. Courtens, Phys. Rev. Lett, 52 (1984) 69.

S.A. Brawer, J. Chem. Phys, 81 (1984) 954.

3 V.H. Schmidt, J.T. Wang, and P.T. Schnackenberg, Jpn. J. Appl. Phys., 24, Suppl. 24-2 (1985) 944.

4 V.H. Schmidt, Ferroelectrics, 72 (1987) 157.

5 E. Matsushita and T. Matsubara, Prog. Theor. Phys., 71 (1984) 235.

6 J. Slak, R. Kind, R. Blinc, E. Courtens, and S. Żumer, Phys. Rev. B, 30 (1984) 85.

7 C.W. Fairall and W. Reese, Phys. Rev. B, 11 (1975) 2066.

8 H.E. Stanley and N. Ostrowsky (Eds.) On Growth and Form, Nijhoff, Boston, 1986.

9 V.S. Dotsenko, J. Phys. C, 18 (1985) 6023.

10 E. Courtens, R. Vacher, and Y. Dagorn, Phys. Rev. B, 33 (1986) 7625.

11 Ya.G. Sinai, Lecture Notes in Physics, 153 (1982) 12.

12 B. Derrida and Y. Pomeau, Phys. Rev. Lett., 48 (1982) 627.

13 R.G. Palmer, D.L. Stein, E. Abrahams, and P.W. Anderson, Phys. Rev. Lett., 53 (1984) 958.

14 M. Randeria, J.P. Sethna, and R.G. Palmer, Phys. Rev. Lett., 54 (1985) 1321. 